\title{
INTERACCIÓN SOCIAL AL SUR DEL COLLASUYU. ALFARERÍA FUNERARIA DEL PERIODO TARDÍO (1400-1536 DC) EN LA CUENCA MAIPO-MAPOCHO
}

\author{
SOCIAL RELATIONS TO THE SOUTH OF COLLASUYU. A STUDY OF \\ FUNERARY POTTERY CONTEXTS OF THE LATE PERIOD (1400-1536 AD) IN \\ THE MAIPO-MAPOCHO BASIN
}

Cristian Dávila, ${ }^{1,2}$, Constanza Cortés ${ }^{1,3}$, Andrea Martínez $z^{1,4}$, Juan Hermosilla ${ }^{1,5}$, Nicole Fuenzalida ${ }^{1,6}$ y Daniel Pavlovic ${ }^{7}$

\begin{abstract}
Aunque existen variados estudios de sitios tardíos en la zona Central de Chile, no hay consenso sobre cómo se habría dado la interacción entre las poblaciones locales Aconcagua y el Inka en esta zona fronteriza del Collasuyu. A partir de la variabilidad alfarera en la zona, se ha propuesto diferentes niveles de interacción. Sin embargo, faltan estudios comparativos sistemáticos que permitan adentrarnos en esta problemática. El presente trabajo busca contribuir en esta línea con el análisis de piezas alfareras provenientes de 17 sitios funerarios de la cuenca Maipo-Mapocho, identificando la emergencia de un nuevo tipo cerámico local y la presencia de vasijas de estilo inkaico que parecieran ser producidas localmente. Al interior de la cuenca, entre las áreas de Chacabuco, Mapocho y Maipo, se reconoce un comportamiento diferencial, lo que podría estar dando cuenta de los distintos niveles de interacción entre las poblaciones locales y el Tawantinsuyu.
\end{abstract}

Palabras claves: Tawantinsuyu, Poblaciones Locales, Referente cultural, Interacción social, contextos funerarios.

Although there are several studies focusing on late sites in Central Chile, there is no consensus about how the interaction between local Aconcagua settlements and the Inka took place in this frontier of the Collasuyu. Through local ceramic variability, different levels of interaction have been proposed; however, there is a lack of systematic comparative studies that allow us to explore this issue. This work aims to contribute in this regard by analyzing ceramic vessels of seventeen funerary sites in the Maipo-Mapocho basin, identifying the emergence of a new local ceramic type and the presence of vessels of Inka style that seem to be locally manufactured. Throughout the basin, between the Chacabuco, Maipo and Mapocho areas, different behaviors are identified, which could account for the varied levels of interaction between the local populations and the Tawantinsuyu.

Key words: Tawantinsuyu, Aconcagua culture, cultural referent, social interaction, funerary context.

El área estudiada se ubica en la frontera sur del Collasuyu, correspondiente a una cuenca hidrográfica de clima mediterráneo con dos afluentes hídricos importantes, el río Maipo y el Mapocho (33³0’ lat. sur). Existe amplia evidencia de la presencia inkaica en esta zona, registrada principalmente a partir de sitios arquitectónicos sobre cerros (Planella y Stehberg 1997; Troncoso et al. 2010; Stehberg 1976a), como también un gran número de contextos funerarios
(Baytelman 1970; Correa et al. 2008; Mostny 1947; Stehberg 1976a, 1976b).

El carácter fronterizo de esta área dentro del Tawantinsuyu, lo situaría con ciertas particularidades desde el registro arqueológico, que se presenta a modo de mosaico, con áreas plenamente incorporadas y otras al margen (González 2000).

A nivel alfarero los estudios de distintos contextos dan cuenta de una heterogeneidad dentro de la cuenca

\footnotetext{
${ }^{1}$ Investigador asociado a Proyecto Fondecyt $\mathrm{N}^{\circ} 1140803$

${ }^{2}$ Ignacio Carrera Pinto 1114-A, depto. 104, Ñuñoa, Santiago, Chile. cristiandavilac@gmail.com

${ }^{3}$ Exequiel Fernández 1129, depto. 44, Ñuñoa, Santiago, Chile. c.cortes.rod@gmail.com

${ }^{4}$ Paicaví 51, casa 1, Valparaíso, Chile. andreamartine@gmail.com

${ }_{5}^{5}$ General Arriagada 884, La Florida, Santiago, Chile. juanghermosilla@ gmail.com

${ }^{6}$ Monseñor Miller No 43, depto. C. Providencia, Santiago, Chile. nicole.fuenzalida.bahamondes@ gmail.com

7 Departamento Antropología, Universidad de Chile. Av. Ignacio Carrera Pinto 1045, Ñuñoa, Santiago, Chile. daniel.pavlovic@gmail.com
}

Recibido: septiembre 2017. Aceptado: junio 2018.

http://dx.doi.org/10.4067/S0717-73562018005002101. Publicado en línea: 2-octubre-2018. 
Maipo-Mapocho, reflejando una producción local y una gran variabilidad para la zona (Sanhueza 2001; Vásquez 1994), que han sido abordados a partir de distintas nomenclaturas, entorpeciendo los estudios comparativos, enfocados principalmente a sitios particulares. Dicha variabilidad ha llevado a proponer distintos niveles de relación entre los locales y el Tawantinsuyu, en un escenario donde la población local habría "negociado" con "otros" su cultura (Cantarutti y Mera 2002), lo que podría responder a diferentes niveles de participación por parte de estos grupos al nuevo orden sociopolítico (Pease 1979).

Actualmente existe consenso en la existencia de estrategias diferenciales por parte del Tawantinsuyu en la interacción con las poblaciones locales, las que varían de acuerdo con las particularidades de cada región y a sus propios intereses (Hayashida 2003), siendo primordiales aquellas relacionadas con el dominio simbólico, correspondiente a la transformación y apropiación de creencias, ritos y espacios ceremoniales locales, articulando de manera distinta las redes sociales previas que estas poblaciones sostenían entre sí y con otras áreas (Pavlovic et al. 2012; Sánchez 2004; Sánchez y Troncoso 2008; Uribe 2000). De esta manera los ritos deberían haber sido cruciales para establecer un nuevo orden, constituyéndose como un importante campo para la representación y manipulación de las relaciones políticas (Dietler 2001).

Este trabajobuscaaproximarse alainteracción entre ambas entidades, Inka y poblaciones locales, durante el periodo Tardío (1400-1536 DC) a partir del estudio de piezas cerámicas halladas en distintos contextos funerarios de la cuenca Maipo-Mapocho, proponiendo una categorización tipológica estandarizada funcional a esta problemática, que permita incluir la variabilidad observada en la incorporación de nuevos referentes culturales durante este periodo, en un área fronteriza del Collasuyu.

\section{Interacciones Sociales y Alfarería en Contextos Funerarios}

La interacción entre el Inka y las poblaciones locales la comprendemos como una relación de poder de producción multiforme, de relaciones de dominación y negociación, integrables en una estrategia en conjunto, donde el poder no sería sólo represivo, sino que también productivo, constructor de conocimientos y realidades (Foucault 1979). Esta relación de poder se establece en todos los ámbitos de la sociedad. Dentro del mundo andino algunos espacios son más adecuados para propiciar esta interacción entre distintos grupos, como por ejemplo, las festividades públicas, que se convertirían en espacios fundamentales para mantener activas las redes de alianzas (Rostworowski 1988). Dentro de esta lógica podemos comprender la funebria como un espacio de congregación pública constituyéndose en un escenario propicio de interacción y alianza (Hayden 2009). De esta forma el Inka interviene en estas instancias, donde entran en juego estas relaciones de poder.

En esta relación existiría resistencia y lucha ideológica, permeando y reproduciendo una forma de estar en el mundo, abriéndose posibilidades para la manipulación y redefinición en diferentes sectores sociales de un grupo. Es decir, existiría un margen en el cual éstos pueden, en cierta medida, modificar la realidad propuesta por el sistema impuesto (Troncoso 2001), surgiendo alternativas en las que se aprecia la agencia de los grupos subalternos.

De esta manera, los grupos culturales generarían distinciones de sí mismos a partir de las interacciones generadas con otros. A la larga, estas constantes interacciones no conducirían necesariamente a una eliminación del sistema social (Barth 1976), sino que las diferencias entre ellos podrían persistir a pesar del contacto constante, e inclusive de la interdependencia. Ahora bien, es de esperar que en estas interacciones sus diferencias disminuyan, pues es necesario una congruencia de códigos y valores, o que se generen instancias en las cuales éstas se puedan establecer (Hodder 1982). Tal es el caso de los ritos funerarios, que constituyen lugares propicios para mantener y establecer relaciones de alianza entre distintas entidades sociales, las que muchas veces se encuentran mediadas a través de la redistribución y comensalismo en fiestas asociadas al ritual, con una fuerte carga de ostentación (Hayden 2009). Así, los rituales funerarios se configuran como procesos complejos en donde convergen diferentes aspectos de sus participantes. Comprendiendo las prácticas mortuorias como "significativamente constituidas" (Hodder 1982), éstas serían elegidas por actores en relación a una creencia específica. De esta manera, el comportamiento mortuorio refleja la cosmovisión de una sociedad más que su organización práctica. Las prácticas asociadas a la funebria pueden ser vistas como resultado de una serie de decisiones ideológicas, políticas, identitarias y económicas, que pueden ser manipuladas para legitimar un orden social que se requiere establecer o perpetuar (Parker 1993), así la esfera funeraria durante el periodo Tardío en la cuenca del Maipo-Mapocho, se convierte en un aspecto privilegiado para adentrarse en las interacciones y negociaciones establecidas entre las poblaciones locales y el Tawantinsuyu.

La alfarería se constituye como el elemento material más representado, actuando como portadora de significados tanto en el consumo, almacenaje y preparación de alimentos, como en el conjunto ofrendado 
en entierros, teniendo la capacidad de intervenir en las interacciones sociales. Además, puede constituirse como un bien de prestigio con acceso restringido y/o generar un código socialmente entendido por un sector de la sociedad o por grupos étnicos determinados (Csikszentmihalyi y Rochberg-Halton 1981). En el ritual mortuorio inkaico, el repertorio cerámico tuvo un papel preponderante, dentro del cual las vasijas poseen un rol político simbólico importante (Bray 2003, 2004), donde la agencia social del Inkanato estaría distribuidas en estas piezas (Gell 1998).

Por otro lado, la producción alfarera implica la toma de decisiones arbitrarias, condicionadas por el contexto sociocultural en el cual los agentes aprenden dichas prácticas (Dietler y Herbich 1998). De este modo, el artesano se apropia de los referentes existentes en su entorno para otorgar un nuevo contenido, cuya inteligibilidad es depositada en los esquemas que constituyen el artefacto (Gallardo et al. 2011). En este sentido, el estilo alfarero sería forzosamente reactivo y siempre susceptible al contexto, construido necesariamente en relación a otros estilos (DeBoer 1990). Por este carácter dialogante y activo, se propone utilizar el concepto de "referente cultural", para distinguir atributos cerámicos (morfológicos, decorativos y tecnológicos) en una determinada producción alfarera, que se relacionan con otros estilos exponentes de tales atributos. Así se busca conceptualizar la presencia de elementos procedentes de otros estilos cerámicos al interior de un determinado estilo, incorporados en la producción en forma de alusión, interpretación, imitación y/o recreación, cumpliendo distintas funciones de acuerdo a la situación socio-histórica particular en la que se desenvuelve el objeto cerámico. En consecuencia, se trata de un recurso en la producción del alfarero, que puede o no ser consciente o voluntario (Fuenzalida 2014).

\section{Muestra, Metodología y Propuesta Tipológica}

Se realizaron análisis sistemáticos de conjuntos cerámicos provenientes de 17 sitios funerarios de la cuenca del Maipo-Mapocho, totalizando 414 piezas completas. Principalmente corresponden a entierros en fosa, sin embargo, se registran algunos casos con mayor inversión de trabajo en funebria, incluyendo estructuras abovedadas, estructuras de piedras y fosas con estructuras, además de una gran variabilidad de ofrendas asociadas, especialmente alfarería (Tabla 1).

La muestra de estudio se subdivide en tres áreas dentro de la cuenca, definidas a partir de la

Tabla 1. Muestra alfarera de análisis según sitio, tipo de entierro y unidades mortuorias (s/r: sin referencia).

Sample of analyzed vessels according to site, type of burial and mortuary units (w/r: without reference).

\begin{tabular}{|c|c|c|c|c|}
\hline Zona & Sitio & Tipo de entierro & $\mathrm{N}^{\circ}$ Unidades mortuorias & $\mathrm{N}^{\circ}$ Vasijas \\
\hline \multirow{5}{*}{ Chacabuco } & Chacabuco & $\mathrm{s} / \mathrm{r}$ & 4 & 6 \\
\hline & El Almendral & Señalizadas por clastos & 5 & 4 \\
\hline & Familia Álvarez & $\mathrm{s} / \mathrm{r}$ & $\mathrm{s} / \mathrm{r}$ & 7 \\
\hline & Los Baños 133 & $\mathrm{~s} / \mathrm{r}$ & $\mathrm{s} / \mathrm{r}$ & 7 \\
\hline & Pablo Carvajal & Fosa & 3 & 43 \\
\hline \multirow{9}{*}{ Mapocho } & Parcela 24 & Fosa con estructura & 1 & 7 \\
\hline & Quilicura 1 & Fosa & 5 & 58 \\
\hline & Lenka Franulic & Fosa & 3 & 8 \\
\hline & Estación Quinta Normal & Fosa & 13 & 18 \\
\hline & La Reina & Estructura abovedada & 5 & 58 \\
\hline & La Reina Cantera & $\mathrm{s} / \mathrm{r}$ & $\mathrm{s} / \mathrm{r}$ & 3 \\
\hline & Mall plaza Los Dominicos & $\mathrm{s} / \mathrm{r}$ & 1 & 1 \\
\hline & Marcoleta & Estructura abovedada & 1 & 13 \\
\hline & Talleres y Cocheras & Señalizadas por clastos & 6 & 9 \\
\hline \multirow{3}{*}{ Maipo } & Nos & Fosa & \pm 30 & 103 \\
\hline & Sector Chena & $\begin{array}{l}\text { Fosa } \\
\text { Estructura de piedra canteada y } \\
\text { cantos rodados }\end{array}$ & 22 & 49 \\
\hline & Los Jazmines & Fosa & 13 & 20 \\
\hline & & Total & & 414 \\
\hline
\end{tabular}


concentración espacial de los sitios, coincidiendo con diferencias geográficas y ambientales. La cuenca del Maipo-Mapocho puede dividirse hidrográficamente en dos áreas. A su vez, en la subcuenca del río Mapocho encontramos los sectores que hemos definido como Chacabuco y Mapocho.

El área de Chacabuco, correspondiente a los sectores de Colina y Lampa actualmente, se caracteriza por presentar cursos hídricos de régimen pluvial de caudal bajo y/o estacional. Esto determina un paisaje de quebradas, valles angostos, amplias llanuras de escasa pendiente y ambiente semiárido, dominado por vegetación xerófita y gramínea, que solo en las quebradas y laderas de umbría presenta formaciones vegetacionales arbóreas más densas.

El sector de Mapocho por su parte, perteneciente la zona donde se emplaza la ciudad de Santiago, presenta características similares, sin embargo, destacan afluentes que nacen en nieves preandinas, destacando el curso del rio Mapocho que otorga un recurso hídrico permanente. Este sector corresponde al lugar que concentra la mayor cantidad de contextos funerarios registrados.

Por último, la subcuenca del río Maipo corresponde al área más meridional, caracterizada por sus afluentes de régimen pluvio-nival, alimentados de la alta cordillera y los glaciares. Esto genera un amplio valle fluvial, con mayor disponibilidad de recursos hídricos que la subcuenca adyacente al norte y que además recibe la influencia moderadora de temperaturas procedente del Pacífico. Si bien, esta área presenta una menor cantidad de contextos funerarios, estos dan cuenta de un mayor número de piezas y unidades mortuorias por sitio (Figura 1).

Se trabajó en una propuesta clasificatoria cerámica a nivel: (1) teórico, enfatizando en la idea de interacción y sincretismo entre el Inka y locales, junto a la incorporación de la idea de referentes culturales en la construcción del estilo alfarero (Fuenzalida 2014), y (2) analítico, priorizando en el análisis morfológico y decorativo de las piezas completas. Como base se empleó la clasificación de Calderari y Williams (1991) debido a su utilización en otras áreas del Tawantinsuyu, pero generando una mayor distinción entre categorías, considerando aspectos antes descritos. Así tenemos:

\section{Alfarería de estilo Inka}

- Inka Local: vasijas que responden a morfología y decoración cuzqueña con diferencias dadas por la manufactura local, principalmente a nivel de proporciones y decoraciones (colores, ejecución de trazos y estructura de diseño). Si bien, esta definición es similar a la de Inka Provincial, se ha preferido utilizar el concepto "local" puesto que no hay evidencia material que indique la existencia de talleres de mitimaes de especialistas cerámicos con control estatal, propios del concepto de Provincia.

- Inka Mixto: vasijas que combinan elementos cuzqueños y no cuzqueños en términos morfológicos y decorativos, pero con énfasis en el diseño morfofuncional inkaico. Es decir, que a nivel formal no cumplen necesariamente con los elementos constitutivos de las vasijas cuzqueñas, pero aluden a éstas, y a nivel decorativo presentan elementos mixtos.

\section{Alfarería de estilo Local}

- Local Aconcagua: estilo Local pre-inkaico propio de la cultura Aconcagua, entendido a partir de la definición de Massone (1978), considerando los tipos Aconcagua Salmón, Aconcagua Rojo Engobado y vasijas monocromas con formas reconocidas desde tiempos preinkaicos.

- Local Fase Inka: vasijas que forman parte de las tradiciones alfareras locales con transformaciones relacionadas con el nuevo contexto socio-político y la proliferación de nuevos referentes. Las transformaciones son a nivel decorativo y morfológico respecto de la alfarería Aconcagua, a través del surgimiento de nuevos elementos (ángulo inscrito, asa labio adherida, etc.) y la incorporación de otros referentes foráneos (Inka, Diaguita, Copiapó, Viluco, Paya).

\section{Alfarería de estilo Diaguita}

- Vasijas que presentan forma y decoración Diaguita (González 2013), tanto a nivel de elementos decorativos, motivos, estructura y disposición del diseño.

Para el análisis de piezas completas fueron considerados principalmente dos elementos: (1) adscripciones estilísticas y/o referentes culturales, evaluando atributos formales y decorativos, y (2) caracterización de la muestra en términos de variabilidad, considerando rasgos decorativos, morfométricos y funcionales, basándose en las clasificaciones propuestas por Shepard (1956).

El análisis decorativo se orientó a caracterizar aquellos tipos cerámicos que presentan mixtura, es decir, Inka Mixto y Local de Fase Inka, ya que los tipos Inka Local, Local Aconcagua y el estilo Diaguita han sido definidos con elementos decorativos propios. Los referentes decorativos identificados en la muestra son de origen Diaguita (González 2013), Inka (Fernández 1971), Copiapó (Garrido 2007), La Paya (Calderari 1988), Aconcagua (Massone 1978) y Local Tardío (diseños locales que surgen durante el periodo Tardío). Fueron consideradas únicamente vasijas con motivos decorativos, descartándose de esta parte del análisis las vasijas Monocromas y Rojas Engobadas. 


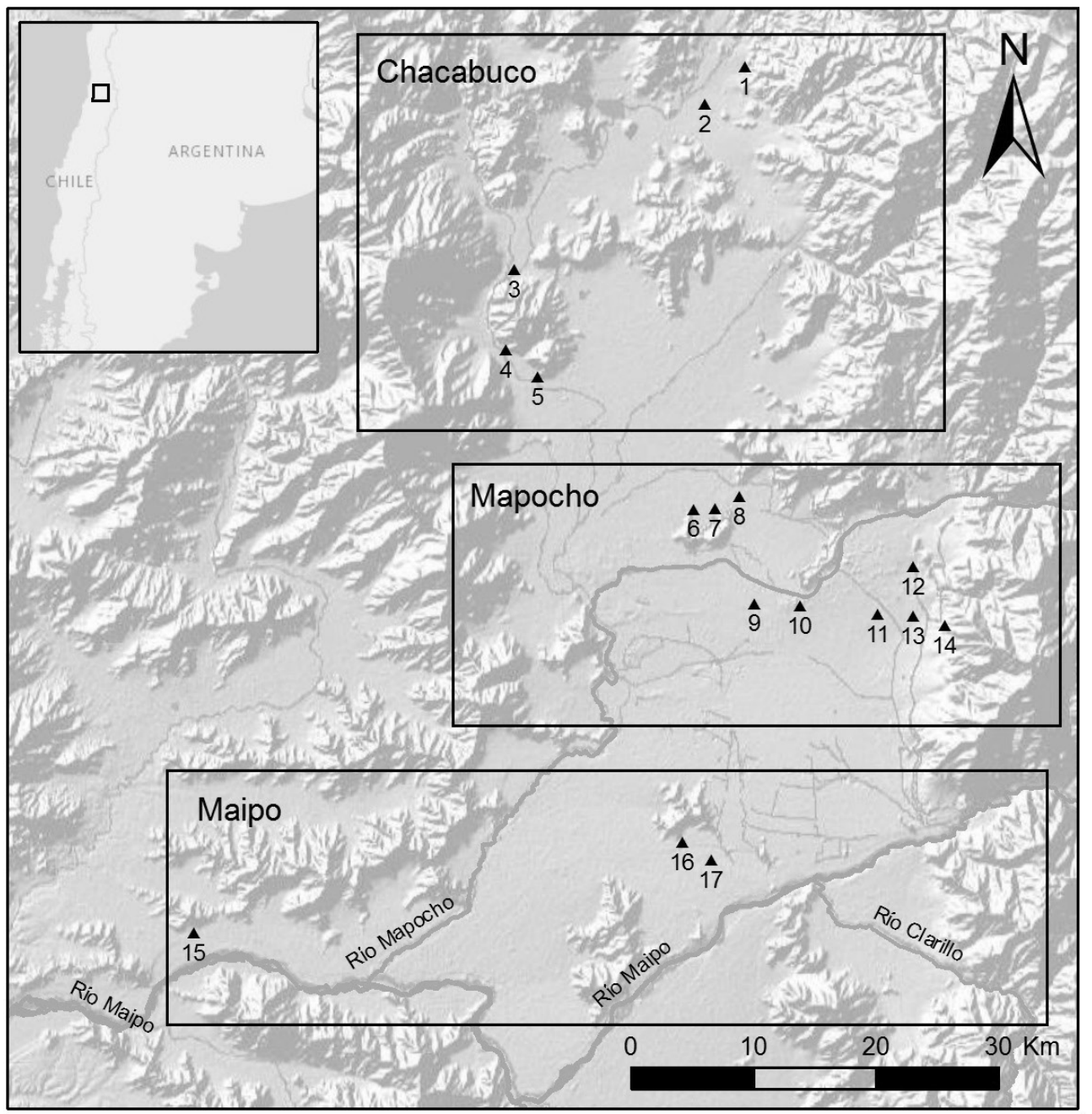

Simbología

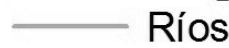

- Sitios Arqueológicos

1: Los Baños 133, 2: Chacabuco, 3: Almendral, 4: Familia Álvarez, 5: Pablo Carvajal, 6: Quilicura 1, 7: Parcela 24, 8: Talleres y Cocheras, 9: Estación Intermodal, 10: Marcoleta, 11: Lenka Franulic, 12: Mall Plaza Los Dominicos, 13: La Reina Cantera, 14: La Reina, 15: Los Jazmines, 16: Chena, 17: Nos.

Figura 1. Sitios funerarios tardíos de la cuenca Maipo-Mapocho.

Late funerary sites of the Maipo-Mapocho basin. 


\section{Resultados}

\section{Adscripción tipológica de la alfarería funeraria tardía}

El análisis revela un predominio del conjunto cerámico de estilo Local por sobre el de estilo Inka, destacando lo Local Fase Inka y lo Inka Mixto respectivamente. Además, se aprecia un porcentaje muy bajo del estilo Diaguita (Tabla 2).

A nivel general, observamos que comparativamente la zona del Mapocho tiene una mayor representación del estilo Inka por sobre el estilo Local, mientras que en las áreas del Maipo y Chacabuco esta relación es inversa. En cuanto al estilo Inka, las piezas Inka Mixto son más abundantes que las Inka Local en todos los sectores. Por su parte, el estilo Local está representado por una mayor frecuencia del tipo Local Fase Inka hacia el sur de la cuenca, mientras que, hacia el norte, hay una mayor preponderancia de Local Aconcagua, aunque siempre con una baja representación.

\section{Formas cerámicas tardías del Maipo-Mapocho}

Dentro de las vasijas de estilo inkaico (Figura 2a, b, c, y f), se reconoce una leve discordancia en cuanto a la proporción de categorías propuesta para las provincias del Tawantisuyu, caracterizado por un predominio de aríbalos, seguido por platos y ollas en pedestal (Bray 2003, 2004). En esta región, los platos son más frecuentes que los aríbalos, mientras que las ollas en pedestal son bastante escasas, a diferencia de la alta recurrencia de aysanas, especialmente en el sector de Maipo y Chacabuco (Tabla 3), forma que en el resto del Tawantinsuyu posee baja representación (Bray 2004). En este sentido, se propone como kit básico inkaico para la cuenca Maipo-Mapocho, en orden de preponderancia, platos, aríbalos y aysanas.

El aríbalo se ha vinculado con el almacenamiento, transporte y servicio de chicha. Su alta presencia en esta área periférica reafirmaría la idea de un énfasis mayor a la distribución de chicha por parte del Inka a las poblaciones locales de las distintas regiones del Tawantinsuyu por sobre el consumo en el área central (Bray 2003, 2004). Ahora bien, la gran mayoría de los aríbalos registrados en este estudio no corresponden exactamente a la forma cuzqueña (Fernández 1971; Matos 1999), pues varios no presentan asas en suspensión, ni protuberancia en el sector superior del cuerpo, ni base ápoda, y sus puntos de quiebres en el cuerpo son suaves o inexistente. Consideramos que estas modificaciones responden al carácter dialogante y activo del estilo dentro del contexto local, donde la estructura de sus atributos morfológicos, sin precedentes locales, responde a lo inkaico y cuya función, a partir de estudios de carporrestos, se asocia a la bebida fermentada (Belmar et al. 2015).

Para el plato se ha propuesto una funcionalidad asociada al consumo de alimentos principalmente sólidos, dada su forma baja (Bray 2003), mientras que las aysanas servirían para servir chicha o agua (Matos 1999), y transportar líquidos en pequeñas cantidades (Fernández 1971), complementando la funcionalidad del aríbalo. Así, estos tres principales tipos de formas cerámicas de referente inkaico representados

Tabla 2. Frecuencia absoluta y relativa de vasijas según adscripción tipológica y área de procedencia.

Absolute and relative frequencies of vessels according to typological ascription and area of origin.

\begin{tabular}{|c|c|c|c|c|c|c|c|c|}
\hline \multirow[t]{2}{*}{ Adscripción Tipológica } & \multicolumn{6}{|c|}{ Área } & \multicolumn{2}{|c|}{ Total } \\
\hline & $\mathrm{N}$ & $\%$ & $\mathrm{~N}$ & $\%$ & $\mathrm{~N}$ & $\%$ & $\mathrm{~N}$ & $\%$ \\
\hline Estilo Inka & 28 & 41,8 & 87 & 49,7 & 68 & 39,5 & 183 & 44,2 \\
\hline Inka Local & 6 & 9,0 & 30 & 17,1 & 17 & 9,9 & 53 & 12,8 \\
\hline Inka Mixto & 22 & 32,8 & 56 & 32 & 50 & 29,1 & 128 & 30,9 \\
\hline Indeterminado & - & - & 1 & 0,6 & 1 & 0,6 & 2 & 0,5 \\
\hline Estilo Local & 34 & $\mathbf{5 0 , 7}$ & 74 & 42,3 & 94 & 54,7 & 202 & 48,8 \\
\hline Local Fase Inka & 22 & 32,8 & 51 & 29,1 & 83 & 48,3 & 156 & 37,7 \\
\hline Local Aconcagua & 12 & 17,9 & 23 & 13,1 & 10 & 5,8 & 45 & 10,9 \\
\hline Indeterminado & - & - & - & - & 1 & 0,6 & 1 & 0,2 \\
\hline Diaguita & 1 & 1,5 & 6 & 3,4 & 4 & 2,3 & 11 & 2,7 \\
\hline Indeterminado & 4 & 6 & 8 & 4,6 & 6 & 3,5 & 18 & 4,3 \\
\hline Total & 67 & 100 & 175 & 100 & 172 & 100 & 414 & 100 \\
\hline
\end{tabular}




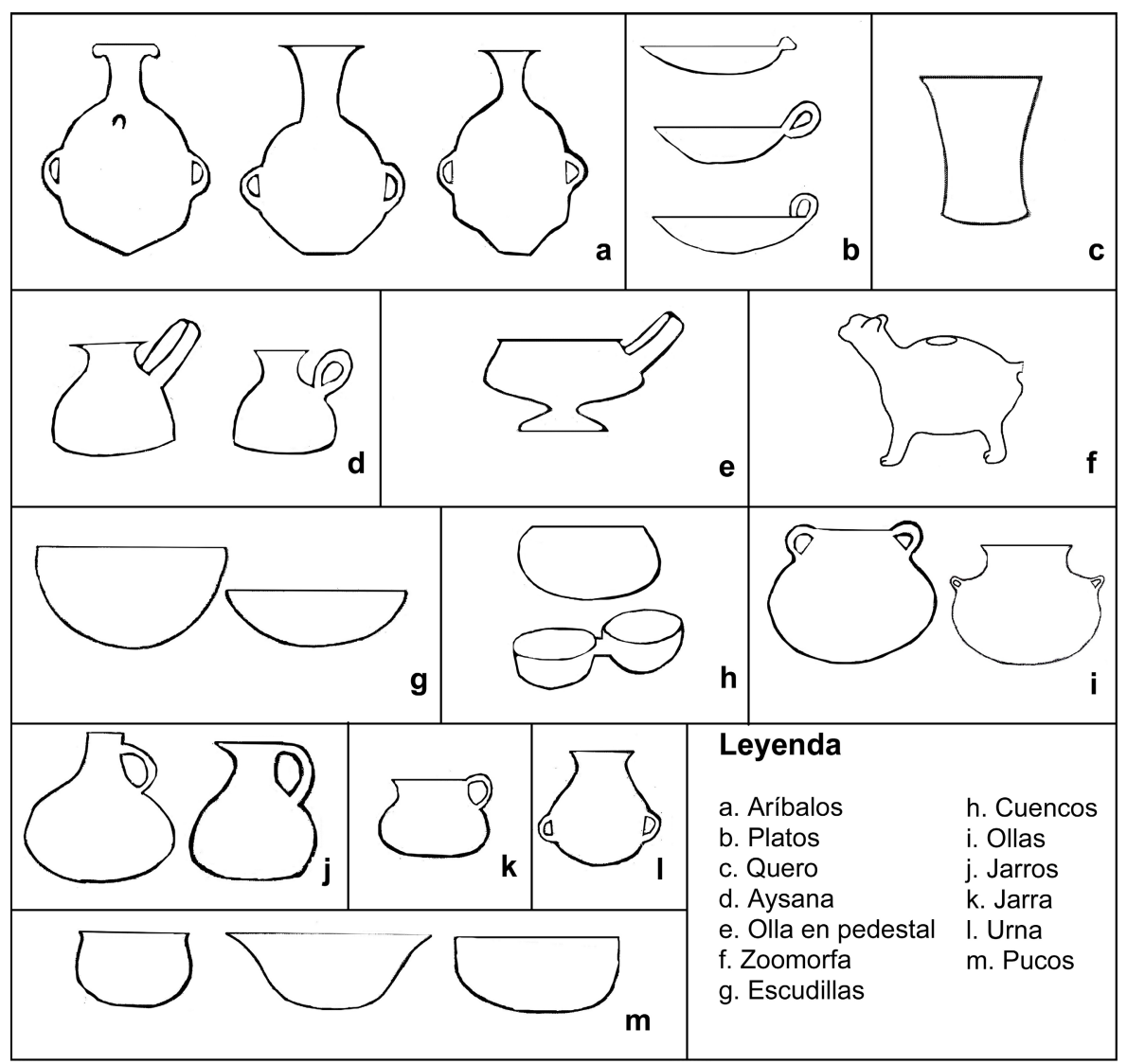

Figura 2. Formas cerámicas tardías en la cuenca Maipo-Mapocho.

Late Ceramic shapes in the Maipo-Mapocho basin.

Tabla 3. Frecuencia absoluta y relativa de vasijas estilo Inka según forma y área de procedencia.

Absolute and relative frequencies of Inka style vessels according to shapes and area of origin.

Forma General

Área

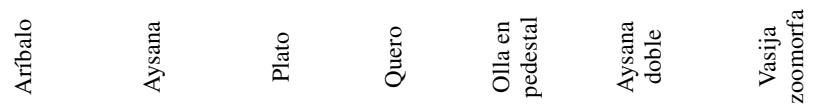

\begin{tabular}{cccccccccc}
\hline \multirow{2}{*}{ Chacabuco } & $\mathrm{N}$ & 10 & 4 & 11 & - & 1 & - & 2 & 28 \\
& $\%$ & 35,7 & 14,3 & 39,3 & - & 3,6 & - & 7,1 & 100 \\
\hline \multirow{2}{*}{ Mapocho } & $\mathrm{N}$ & 31 & 7 & 45 & 2 & 2 & - & - & 87 \\
& $\%$ & 35,6 & 8,0 & 51,7 & 2,3 & 2,3 & - & - & 100 \\
\hline \multirow{2}{*}{ Maipo } & $\mathrm{N}$ & 16 & 12 & 39 & - & - & 1 & - & 68 \\
& $\%$ & 23,5 & 17,6 & 57,4 & - & - & 1,5 & - & 100 \\
\hline \multirow{2}{*}{ Total } & $\mathrm{N}$ & 57 & 23 & 95 & 2 & 3 & 1 & 2 & 183 \\
& $\%$ & 31,1 & 12,6 & 51,9 & 1,1 & 1,6 & 0,5 & 1,1 & 100 \\
\hline
\end{tabular}


en Maipo-Mapocho son asociados directamente al Tawantinsuyu, formando parte integral de las estrategias de legitimación, como equipo culinario de las distintas instancias de negociación y comensalismo político, siendo un agente importante en el discurso inkaico (Bray 2003). Así, esta alfarería actuaría como objeto material de la ideología inkaica, adquiriendo una importante carga simbólica (Williams 2008), incluso sin la necesidad de la presencia fáctica de personeros del inkanato. A través de estos objetos, el Tawantinsuyu estaría presente.

También se reconocen otras formas de estilo inkaico en menor cantidad. Entre ellas se observa una escasa presencia de ollas en pedestal (en Chacabuco y Mapocho), cuya función se vincula a la preparación de alimentos (Bray 2003). En contraposición, se observa una alta presencia de ollas de estilo local en los tres sectores. También se registra en el sector de Chacabuco dos vasijas zoomorfas inkaicas asociadas al almacenaje de psicotrópicos (Gentile 1984-1985) (Tabla 3). Por su parte, en el Mapocho cabe destacar la presencia de queros, objetos con importante valor simbólico en el discurso socio-político del Inka, ya que su particular forma se asemejaría conscientemente a los vasos rituales utilizados por desarrollos culturales andinos previos (Cummins 2015), siendo muy escasos en la región y en Chile Central (Pascual et al. 2018).

Por otro lado, en el estilo Local (Figura 2g, h, i, j, k y l), predominan las vasijas del tipo Local Fase Inka, que presentan un comportamiento distinto a lo Local Aconcagua, reconociéndose una mayor variabilidad de formas de este tipo en el sector de Maipo. Destaca dentro de este grupo la alta presencia de escudillas tanto en Mapocho como en Maipo, a diferencia de
Chacabuco, sector donde predominan los cuencos. Ambas formas pueden ser vinculadas al consumo de alimentos líquidos y/o sólidos. Especial atención suscitan los jarros que se presentan en porcentajes relativamente similares en las tres zonas de la cuenca (Tabla 4). Por otro lado, las ollas, que tradicionalmente han sido asociadas a la preparación de alimentos se registran en proporciones similares en Chacabuco y Mapocho, mientras que en Maipo son más escasas (Tabla 4).

En cuanto a lo Local Aconcagua se aprecia una menor variabilidad de formas, predominando las escudillas en las tres áreas. Aunque destaca en Chacabuco una mayor representación de cuencos que en las otras áreas, en desmedro de la representación de escudillas. Por otro lado, la olla Local Aconcagua sólo se registra en Maipo, coincidente con la menor proporción de las ollas de estilo Local Fase Inka (Tabla $5)$.

Por último, el escaso material de estilo Diaguita (Figura $2 \mathrm{~m}$ ) corresponde principalmente a pucos compuestos y jarros asimétricos, además de dos urnas en el sector de Mapocho. En Chacabuco sólo se reconoció puco compuesto (Tabla 5).

\section{Referentes Decorativos}

Se identificaron 14 categorías de referentes decorativos y combinaciones que se presentan agrupadas en la Figura 3, con una preponderancia del referente Inka (Figura 4a), tanto de manera individual como en combinación con elementos Local (Figura 4d). En menor proporción se registran las combinaciones Inka con Diaguita, e Inka con otros motivos.

Tabla 4. Frecuencia absoluta y relativa de vasijas Local Fase Inka según forma y área de procedencia.

Absolute and relative frequencies of Inka-phase Local style vessels according to shape and area of origin.

Forma General

\begin{tabular}{|c|c|c|c|c|c|c|c|c|c|c|c|c|}
\hline \multicolumn{2}{|l|}{ Área } & \multirow{2}{*}{$\begin{array}{l}\stackrel{0}{0} \\
\stackrel{\Xi}{\Xi} \\
\\
8\end{array}$} & \multirow{2}{*}{ 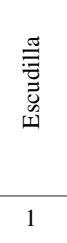 } & \multirow{2}{*}{$\begin{array}{l}\stackrel{\mathbb{E}}{\mathrm{E}} \\
2\end{array}$} & \multirow{2}{*}{ 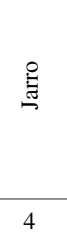 } & \multirow{2}{*}{ 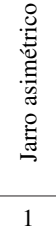 } & \multirow{2}{*}{$\begin{array}{c}\frac{\tilde{\sigma}}{\overline{0}} \\
4\end{array}$} & \multirow{2}{*}{ 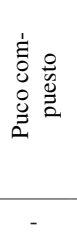 } & \multirow{2}{*}{ 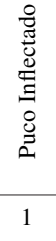 } & \multirow{2}{*}{$\begin{array}{l}\stackrel{\Xi}{\Xi} \\
5 \\
-\end{array}$} & \multirow{2}{*}{$\begin{array}{c}\stackrel{\infty}{0} \\
\stackrel{0}{0} \\
1\end{array}$} & \multirow{2}{*}{$\begin{array}{c}\text { Total } \\
\\
22\end{array}$} \\
\hline & $\mathrm{N}$ & & & & & & & & & & & \\
\hline Cllacabuco & $\%$ & 36,4 & 4,5 & 9,1 & 18,2 & 4,5 & 18,2 & - & 4,5 & - & 4,5 & 100 \\
\hline \multirow{2}{*}{ Mapocho } & $\mathrm{N}$ & 7 & 16 & 4 & 11 & - & 8 & - & 4 & - & 1 & 51 \\
\hline & $\%$ & 13,7 & 31,4 & 7,8 & 21,6 & - & 15,7 & - & 7,8 & - & 2,0 & 100 \\
\hline \multirow{2}{*}{ Maipo } & $\mathrm{N}$ & 12 & 30 & 10 & 15 & 4 & 6 & 3 & 1 & 1 & 1 & 83 \\
\hline & $\%$ & 14,5 & 36,1 & 12,0 & 18,1 & 4,8 & 7,2 & 3,6 & 1,2 & 1,2 & 1,2 & 100 \\
\hline \multirow{2}{*}{ Total } & $\mathrm{N}$ & 27 & 47 & 16 & 30 & 5 & 18 & 3 & 6 & 1 & 3 & 156 \\
\hline & $\%$ & 17,3 & 30,1 & 10,3 & 19,2 & 3,2 & 11,5 & 1,9 & 3,8 & 0,6 & 1,9 & 100 \\
\hline
\end{tabular}


Tabla 5. Frecuencia absoluta y relativa de vasijas Local Aconcagua y Diaguita según forma y área de procedencia. Absolute and relative frequencies of Aconcagua and Diaguita Local style vessels according to shape and area of origin.

\begin{tabular}{|c|c|c|c|c|c|c|c|c|c|}
\hline \multirow[b]{2}{*}{ Adscripción } & \multirow[b]{2}{*}{ Área } & \multicolumn{8}{|c|}{ Forma General } \\
\hline & & & $\begin{array}{l}\stackrel{\Xi}{\Xi} \\
\stackrel{\Xi}{\Xi}\end{array}$ & 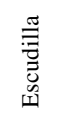 & 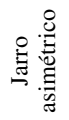 & $\frac{\pi}{\overline{0}}$ & 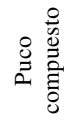 & $\stackrel{\widetilde{\Xi}}{\Xi}$ & Total \\
\hline \multirow{6}{*}{ 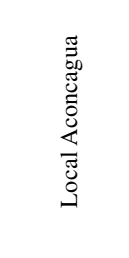 } & \multirow{2}{*}{ Chacabuco } & $\mathrm{N}$ & 4 & 8 & - & - & - & - & 12 \\
\hline & & $\%$ & 33,3 & 66,7 & - & - & - & - & 100 \\
\hline & \multirow{2}{*}{ Mapocho } & $\mathrm{N}$ & 5 & 18 & - & - & - & - & 23 \\
\hline & & $\%$ & 21,7 & 78,3 & - & - & - & - & 100 \\
\hline & \multirow{2}{*}{ Maipo } & $\mathrm{N}$ & 1 & 8 & - & 1 & - & - & 10 \\
\hline & & $\%$ & 10,0 & 80,0 & - & 10,0 & - & - & 100 \\
\hline \multirow{6}{*}{ 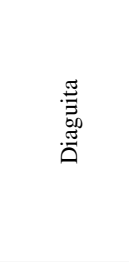 } & \multirow{2}{*}{ Chacabuco } & $\mathrm{N}$ & - & - & - & - & 1 & - & 1 \\
\hline & & $\%$ & - & - & - & - & 100,0 & - & 100 \\
\hline & \multirow{2}{*}{ Mapocho } & $\mathrm{N}$ & - & - & 1 & - & 3 & 2 & 6 \\
\hline & & $\%$ & - & - & 16,7 & - & 50,0 & 33,3 & 100 \\
\hline & \multirow{2}{*}{ Maipo } & $\mathrm{N}$ & - & - & 1 & - & 3 & - & 4 \\
\hline & & $\%$ & - & - & 25,0 & - & 75,0 & - & 100 \\
\hline \multirow{2}{*}{\multicolumn{2}{|c|}{ Total }} & $\mathrm{N}$ & 10 & 34 & 2 & 1 & 7 & 2 & 56 \\
\hline & & $\%$ & 17,9 & 60,7 & 3,6 & 1,8 & 12,5 & 3,6 & 100 \\
\hline
\end{tabular}

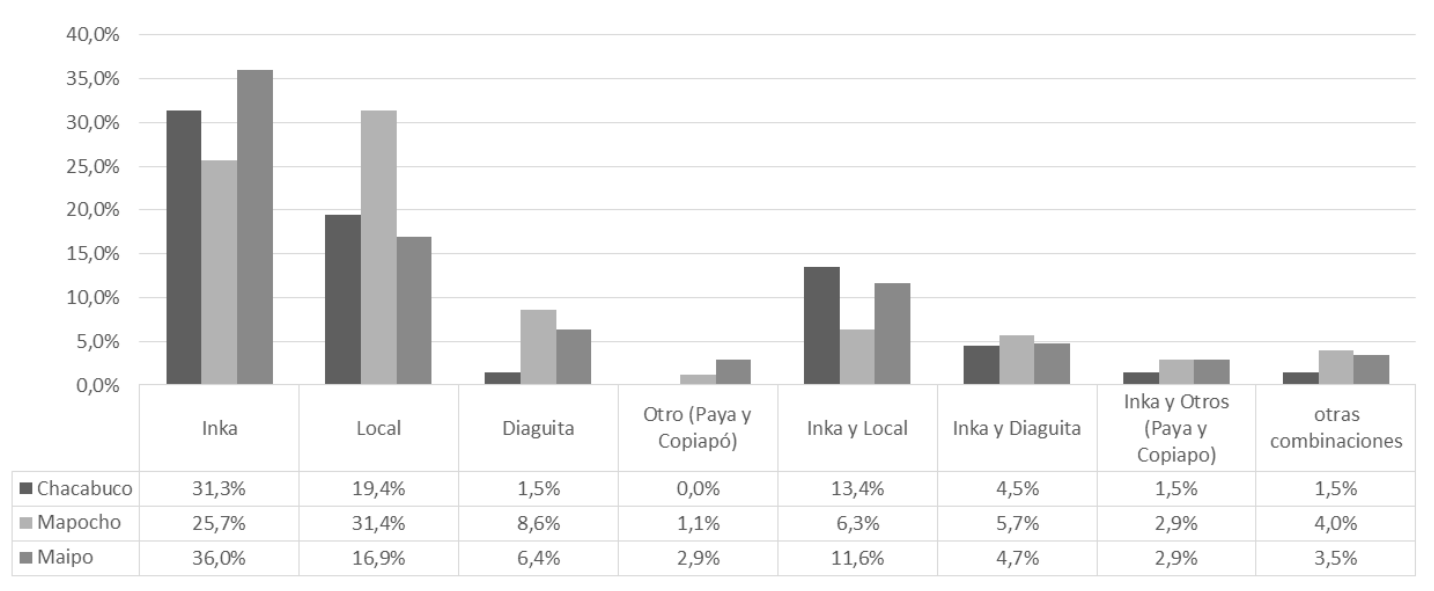

Figura 3. Frecuencia de referentes decorativos según área geográfica.

Decorative referents frequency by geographical area. 


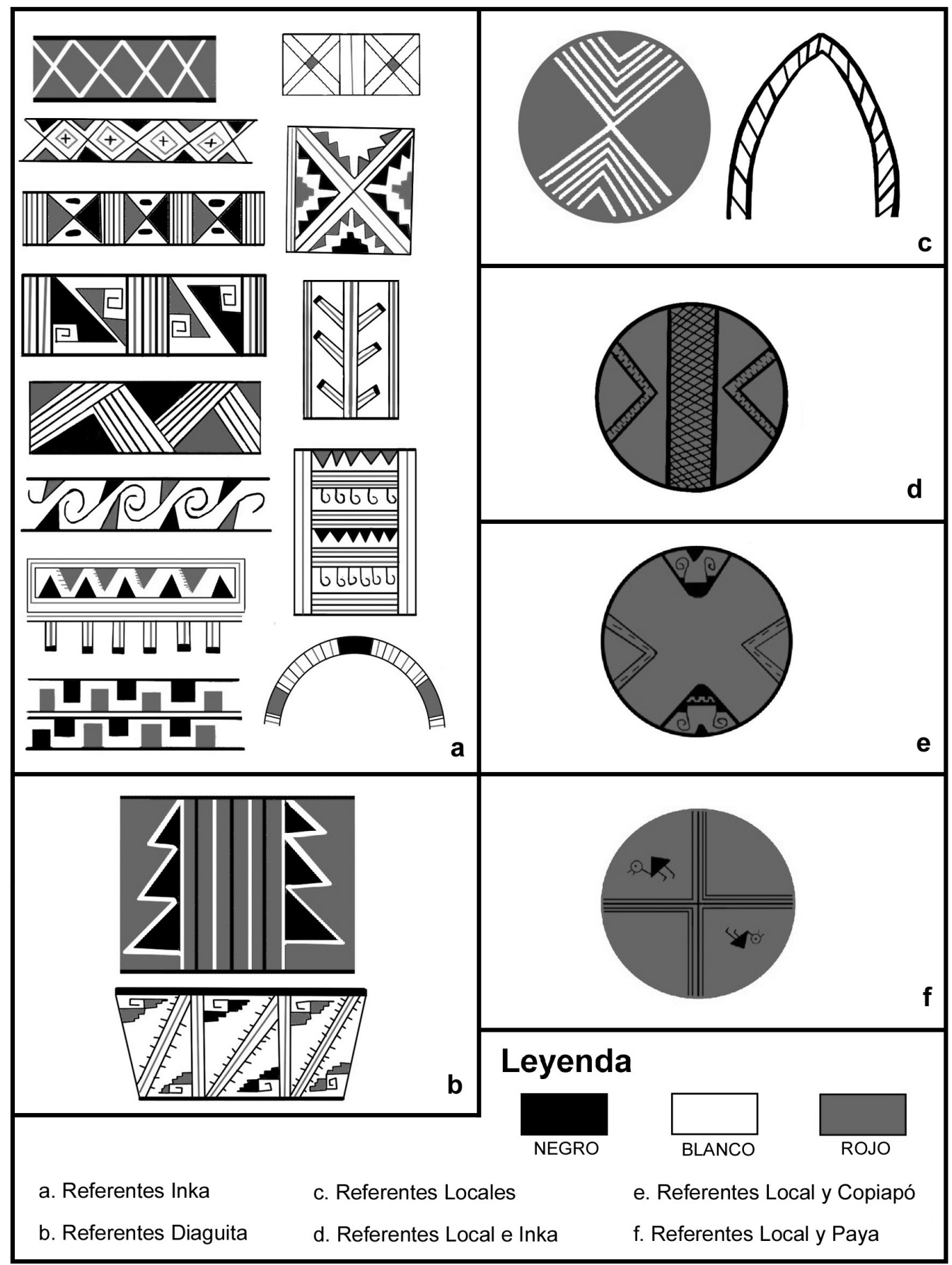

Figure 4. Motivos decorativos frecuentes en la alfarería tardía del Maipo-Mapocho.

Frequent decorative motifs in the late Maipo-Mapocho pottery. 
Por su parte, los Motivos Locales (Figura 4c) varían en cada zona, destacando una mayor presencia en Mapocho. La combinación de Motivos Inka/ Locales se aprecia en las tres áreas, registrando un mayor porcentaje en Chacabuco y Maipo, mientras que en Mapocho esta combinación se presenta en una proporción similar a lo Inka/Diaguita (Figura 3).

Los Motivos Diaguitas (Figura 4b) poseen un porcentaje bastante bajo de representación en las tres áreas estudiadas, siendo casi inexistentes en Chacabuco, situación que aumenta al presentarse en combinación a referentes decorativos inkaicos (Motivos Inka/Diaguitas), registrándose esta situación en las tres áreas (Figura 3).

Otros referentes foráneos identificados corresponden a los motivos Paya (Figura 4f) y Copiapó (Figura 4e), que si bien se presentan exiguamente, se registran en Mapocho y Maipo. La representación de estos motivos aumenta al combinarlo por ejemplo con motivos inkaicos, registrándose en las tres zonas (Figura 3).

De esta forma, a modo general observamos que Chacabuco presenta menor diversidad de referentes decorativos, destacando principalmente referentes inkaicos. Mientras que Mapocho registra la mayor variabilidad de referentes decorativos, y una mayor complejidad, ya que las vasijas pueden presentar la combinación de tres o cuatro referentes en una sola pieza. Además los referentes decorativos Diaguita son más recurrentes que en Chacabuco y Maipo, tanto de manera individual como en combinación con otros elementos decorativos. De esta manera, Mapocho parece ser una zona con mayor presencia de referentes decorativos foráneos, como Paya, Diaguita y Copiapó, siendo el componente Diaguita el más frecuente. A esto se suma que, a la inversa de las otras zonas, el referente decorativo más representado corresponde a lo Local, seguido por lo inkaico.

Por su parte, Maipo registra una variabilidad semejante a la de Mapocho, sin embargo, las proporciones presentes son levemente diferentes, correspondiendo a la zona donde se presenta el más alto porcentaje de elementos decorativos de referente Inka.

\section{Discusiones y Conclusiones}

Dentro de la alfarería tardía de esta región se aprecia una gran variabilidad de referentes formales y decorativos. Dicha variabilidad ha permitido entender estos contextos mortuorios como espacios de interacción y negociación entre el Tawantinsuyu y las poblaciones locales, en los cuales el registro arqueológico correspondería al producto final del ritual funerario y de las actividades realizadas en él. En este sentido, las vasijas cerámicas actuarían como portadoras de significados, mediatizadas por las prácticas fúnebres, sin ser un reflejo directo de la realidad sino, más bien, de una cosmovisión y/o de un orden social que buscaba ser legitimado.

Lamentablemente aún no contamos con fechados que permitan observar si dentro de este mismo periodo existieron variaciones, sin embargo, la muestra analizada otorga información suficiente para proponer un panorama general para estos contextos en las zonas de Chacabuco, Mapocho y Maipo.

Las prácticas funerarias de las poblaciones Aconcagua cambiarían durante el periodo Tardío en esta zona, de entierros simples con escasas ofrendas, y de cementerios de túmulos, registrados sólo en Chacabuco (Falabella et al. 2016), a la incorporación de fosa simple o con arquitectura y estructuras abovedadas o de piedra, a las que se asociaban una gran cantidad y variedad de ofrendas. En este periodo también surgiría un nuevo estilo cerámico, denominado "Local Fase Inka", que mantendría elementos de las tradiciones alfareras locales Aconcagua, presentando cambios a nivel morfológico y decorativo. Esta aparición daría cuenta del impacto de la presencia del Inka, transformando la manufactura alfarera tradicional, pero manteniendo elementos propios de la producción local. A su vez, se mantendrían modos de hacer tradicionales, coexistiendo lo Local Aconcagua con estos nuevos saberes. Esta situación puede interpretarse como: (a) un segmento de la población local, más cercana al Inka, habría iniciado una nueva forma de producir cerámica, mientras que otro habría mantenido sus formas y técnicas tradicionales, coherente con lo propuesto por Cantarutti y Mera (2002); y (b) este nuevo tipo alfarero estaría relacionado a contextos ceremoniales específicos, en este caso el mortuorio, conformando parte del repertorio de los mismos productores alfareros locales. Ambos lineamientos deben contrastarse con el análisis de contextos habitacionales, para comprobar si existe una similitud con las esferas domésticas. En cualquier caso, se observa una fuerte agencia local, donde estos deciden qué elementos integrar con o sin modificaciones y cuáles mantener.

Ambas esferas, local e Inka, habrían interactuado sin mayor mediación de alguna entidad foránea, evidenciándose una significativa de mixtura de ambos referentes en las piezas alfareras. Asimismo, el conjunto alfarero de Estilo Inka, no respondería exactamente a los cánones cuzqueños, registrándose mixtura con referentes locales a nivel formal y decorativo, lo que denotaría posiblemente una manufactura local. Por otro lado, se reconoce también la inclusión de elementos foráneos a nivel decorativo de otras regiones del Collasuyu (Diaguita, Paya y Copiapó). Esto es coherente desde el discurso iconográfico, con estrategias de integración en 
contextos de ceremonias comunitarias de esta nueva región al Tawantinsuyu, como un agente importante en las negociaciones entre el Inka y las poblaciones locales (Pavlovic et al. 2012). Cabe recordar que estos referentes culturales observados a nivel alfarero no necesariamente dan cuenta de la llegada de nuevos grupos.

Las vasijas de Estilo Diaguita se registran sólo en contextos puntuales (Pablo Carvajal, Quilicura 1, La Reina, Estación Quinta Normal y Nos), tal como en otros tipos de sitios arqueológicos (Cornejo y Saavedra 2018); pero al situarlo comparativamente en un contexto regional, su frecuencia resulta ser nula fuera de los sitios mencionados, situación que pone en cuestión la idea de mitimaes diaguitas mediando relaciones entre las poblaciones Aconcagua y el Inka. De esta manera, la presencia de elementos iconográficos foráneos respondería, más bien, a que el Inka facilitaría la transmisión de ciertos elementos y/o referentes que son afines a su discurso, provenientes de comunidades ya integradas a la esfera del Tawantinsuyu, en este caso los diaguitas. Lo que también se aprecia en relación a lo Copiapó y Paya, a nivel decorativo, en la alfarería Diaguita en su zona de origen (norte semiárido) y en los conjuntos cerámicos del Maipo-Mapocho.

El conjunto cerámico tardío se comporta de manera diferencial en los distintos sectores de la cuenca Maipo-Mapocho, lo que indicaría diferentes dinámicas de interacción entre el Inka y las poblaciones locales. Esto podría relacionarse con la diversidad de los grupos Aconcagua en momentos previos a la llegada del Inka. Por una parte en Chacabuco, diversos estudios, tanto a partir de la funebria como en espacios habitacionales dan cuenta de un importante enclave poblacional durante el periodo Intermedio Tardío, donde la Cultura Aconcagua estaría organizada en comunidades corresidenciales que se congregan a través de sus espacios funerarios, consistentes en cementerios de túmulos (Durán 1979; Durán et al. 1999; Falabella et al. 2016; Gaete 1993; Pavlovic et al. 1998a; Sánchez 1995). Por otra parte, en el área del Maipo, el patrón de asentamiento de la cultura Aconcagua es más disperso, destacando la ausencia de cementerios de túmulos (Falabella et al. 2001; Pavlovic y Troncoso 2001; Pavlovic et al. 1998b; Planella y Stehberg 1997).

Es esperable que esta diferenciación también existiera en el espacio intermedio de la cuenca, Mapocho, donde si bien no existe una caracterización específica del periodo previo, durante el periodo Tardío se observa un comportamiento diferente, con una mayor variabilidad de tipos cerámicos, referentes formales y decorativos, evidenciándose además una mayor presencia de vasijas de estilo inkaico en relación a las otras áreas de la cuenca. Aunque esta situación se podría asociar con la importancia política inferida para la zona del Mapocho, como centro administrativo (Stehberg y Sotomayor 2012) o político (Cornejo y Saavedra 2018), consideramos que antes de confirmar esta hipótesis, es necesario considerar dos factores: (1) un sustrato importante que sustenta estas propuestas se relacionan con la alta densidad de material tardío, principalmente funerario, reconocida para esta área, que puede estar fuertemente sesgada por la extensiva remoción del subsuelo producida por el desarrollo urbano desde tiempos de contacto hasta la actualidad; (2) a pesar de la alta intervención antrópica de esta zona, no se han identificado cerros sacralizados con estructuras cercanos al valle en Mapocho, a diferencia de Maipo, por lo que se podría proponer de manera preliminar, que los rituales mortuorio corresponderían a los principales eventos de congregación y negociación política del Inka en el Mapocho, mientras que en el Maipo estos tipos de eventos se asociarían más a los cerros sacralizados y no solo a los espacios funerarios.

De este modo, es posible que las diferencias previas entre las poblaciones locales de la cuenca fueran determinantes en las estrategias diferenciales de dominio simbólico establecidas por el Tawantinsuyu, que se habrían adecuado a las dinámicas particulares de cada región (Hayashida 2003). Sin embargo, no hay que obviar que estas diferencias también deben responder al desarrollo de las interacciones del Inkanato con las poblaciones locales durante este período. Así, aunque este estudio espera generar un aporte al avance de la discusión sobre el periodo Tardío de Chile Central, sabemos que es necesario el contraste de esta información, de forma sistemática, con otros tipos de contextos arqueológicos, para visibilizar el complejo proceso de interacción sociocultural e ideológico generado entre las poblaciones Aconcagua y el Tawantinsuyu, donde habrían entrado en contacto dos lógicas o cosmovisiones con puntos tanto de congruencia como de notoria divergencia, generando cambios en ambas entidades respectivamente, quedando plasmado en la materialidad cultural.

Agradecimientos: La realización de este artículo no podría haber sido posible sin el financiamiento del Proyecto FONDECYT 1140803 y el apoyo de su equipo de trabajo, asimismo de las instituciones que nos permitieron acceder a sus colecciones: Departamento de Antropología de la Universidad de Chile, Museo Nacional de Historia Natural, Museo Regional de Rancagua, Centro de Exhibición Arqueológica Valle de Chicauma y Museo de Colchagua. Agradecemos especialmente las contribuciones de nuestras colegas Elena Aránguiz, Isabella Quiroz, Josefa Ramos y Rodrigo Sánchez. Agradecemos igualmente a los evaluadores anónimos del artículo. 


\section{Referencias Citadas}

Barth, F. 1976. Los Grupos Étnicos y sus Fronteras. La Organización Social de las Diferencias Culturales. Fondo de Cultura Económica, México D.F.

Baytelman, B. 1970. En pleno centro de Santiago un cementerio incaico-español. Viaje 438:12-13.

Belmar, C., L. Quiroz 2015. Informe Arqueobotánico preliminar del sitio Quilicura 1: Análisis de evidencia microfósiles y carporrestos. Informe año I proyecto Fondecyt 1140803.

Bray, T. 2003. Inka pottery as culinary equipment: Food, feasting, and gender in imperial statedesign. Latin American Antiquity 14:1-22.

Bray, T. 2004. La alfarería imperial Inka: una comparación entre la cerámica estatal del área de Cusco y la cerámica de las provincias. Chungara Revista de Antropología Chilena 36 (2):365-374.

Calderari, M. 1988. Estilos cerámicos incaicos de La Paya. Actas del XI Congreso Nacional de Arqueología Chilena, Vol. 2, pp. 151-163. Universidad de Chile, Santiago.

Calderari, M. y V. Williams 1991. Reevaluación de los estilos cerámicos incaicos en el noreste argentino. En El Imperio Inka. Actualización y Perspectivas por Registros Arqueológicos y Etnohistóricos. Comechingonia 9, Número Especial, Vol I: 74-95.

Cantarutti, G. y R. Mera 2002. Alfarería del cementerio Estación Matucana: ensayo de clasificación y relaciones con la cerámica del período Inca de Chile Central y áreas vecinas. Werkén 3:147-170.

Cornejo, L. y M. Saavedra 2018. El Centro Político Inka en el extremo Austral del Tawantinsuyu (Chile Central). Boletín del Museo Chileno de Arte Precolombino 23 (1):133-158.

Correa, I., F. Bahamondes, M. Uribe y C. Solervicens 20072008. Contextos alfareros de interacción social: lo local y lo foráneo en el cementerio inca de Quinta Normal. Revista Chilena de Antropología 19:143-171.

Csikszentmihalyi, M. y E. Rochberg-Halton 1981. The Meaning of Things. Domestic Symbols and the Self. Cambridge University Press, Cambridge.

Cummins, T. 2015. "Inka Art". The Inka Empire: A Multidisciplinary Approach, editado por I. Shimada, pp. 165-196. University of Texas Press, Austin.

DeBoer, W. 1990. Interaction, imitation and communication as expressed in style: the Uyacali experience. En The Uses of Style in Archaeology, editado por M. Conkey y C. Hastorf, pp. 82-104. Cambridge University Press, Cambridge.

Dietler, M. 2001. Theorizing the feasts: Rituals of consumption, commensal, and power in African context. En Feasts, Archaeological and Ethnographic Perspective on Food, Politics, and Power, editado por M. Dietler y B. Hayden, pp. 65-114. Smithsonian Institution Press, Washington D.C. y Londres.

Dietler, M. e I. Herbich 1998. Habitus, techniques, style: an integrate approach to the social understanding of material culture and boundaries. En The Archaeology of Social Boundaries, editado por M. Stark, pp. 222-263. Smithsonian Institution Press, Washington D.C.
Durán, A. 1979. Estudio Arqueológico de un Cementerio de Túmulos Aconcagua Salmón del Sitio El Valle-Chicauma de Lampa. Tesis de grado, Departamento de Antropología, Universidad de Chile, Santiago.

Durán, E., A. Rodríguez y C. González 1999. El paso del Buey: cementerio de túmulos Aconcagua en la cuesta de Chacabuco (Chile Central). Chungara Revista de Antropología Chilena 31 (1):29-48.

Falabella, F., L. Cornejo y L. Sanhueza 2001. Variaciones locales y regionales en la cultura Aconcagua del valle del río Maipo. Actas IV Congreso Chileno de Antropología, Tomo II, pp. 1411-1419.

Falabella, F., D. Pavlovic, M.T. Planella y L. Sanhueza 2016. Diversidad y heterogeneidad cultural y social en Chile Central durante los periodos Alfareros Temprano e Intermedio Tardío (300 años a.C a 1.450 años d.C). En Prehistoria en Chile. desde sus Primeros Habitantes Hasta los Incas, editado por F. Falabella, M. Uribe, L Sanhueza, C. Aldunate y J. Hidalgo, pp. 365-400. Editorial Universitaria, Santiago.

Fernández, J. 1971. Motivos de la Ornamentación de la Cerámica Inca Cuzco. Tomo I y II. Librería Studium ED, Lima.

Foucault, M. 1979. Microfísica del Poder. Ediciones de la Piqueta, Madrid.

Fuenzalida, N. 2014. La Vida en la Muerte: Resistencias e Incanización en la Alfarería Fúnebre de las Comunidades del Curso Medio-Inferior del Aconcagua. Memoria para optar al título profesional de Arqueóloga. Departamento de Antropología, Universidad de Chile, Santiago.

Gaete, N. 1993. R.M.L. 015 "Familia Fernández". Análisis de un contexto Aconcagua atípico en Chile Central. Actas del XII Congreso Nacional de Arqueología Chilena (1991) Tomo II. Temuco. Boletín del Museo Regional de la Araucania 4:249-262.

Gallardo, F., I. Montt, G. Pimentel, M. Sepúlveda, C. Sinclaire y B. Casses 2011. Taller de trabajo. Diseño, estilo e interacción social en arqueología. Proyecto Fondecyt 1070083. Manuscrito en posesión de los autores.

Garrido, F. 2007. El Camélido Sagrado y el Hombre de los Valles: Una Aproximación a la Cultura Copiapó y sus Relaciones a Partir de la Alfarería. Memoria para optar al título de Arqueólogo, Universidad de Chile, Santiago.

Gell, A. 1998. Art and Agency. An Anthropological Theory. Oxford University Press, Oxford.

Gentile, M. 1984-1985. Hulti. Acerca del uso de cierta alfarería Tiwanaku expansivo. Relaciones de la Sociedad Argentina de Antropología XVI ns:205-220.

González, P. 2000. Patrones decorativos de las culturas agroalfareras de la Provincia del Choapa y su relación con los desarrollos culturales de las áreas aledañas (Norte Chico y Zona Central). Actas del XIV Congreso Nacional de Arqueología Chilena, Vol. 2, pp. 191-221. Contribución Arqueológica 5, Copiapó.

González, P. 2013. Arte y Cultura Diaguita Chilena. Simetría, Simbolismo e Identidad. Serie Monográfica de la Sociedad Chilena de Arqueología 2, Santiago.

Hayashida, F. 2003. Leyendo el registro arqueológico del dominio Inka: Reflexiones desde la costa norte del Perú. Boletín de Arqueología PUCP 7:305-319. 
Hayden, B. 2009. Funerals as feasts: why are they so important? Cambridge Archaeological Journal 19 (1):29-52.

Hodder, I. 1982. Symbols in Action: Ehnoarchaeological studies of Material Culture. Cambridge University Press, Nueva York.

Massone, M. 1978. Los Tipos Cerámicos del Complejo Cultural Aconcagua. Memoria para optar al título profesional de Arqueólogo. Departamento de Antropología, Universidad de Chile, Santiago.

Matos, R. 1999. La cerámica Inca. En Los incas. Arte y simbolismo. Colección Arte y Tesoros del Perú. Banco de Crédito del Perú, Lima.

Mostny, G. 1947. Un cementerio incásico en Chile Central. Boletín del Museo Nacional de Historia Natural 23:17-41.

Parker,M. 1993. The Powerful of Dead:Archeological relationships between the Living and Dead. Cambridge Archeological Journal 3 (2):203-229.

Pascual, D., A. Martínez, D. Pavlovic, C. Dávila, C. Cortés, M. Albán y N. Fuenzalida 2018. Queros de cerámica y la presencia del Tawantinsuyu en la cuenca de los ríos Aconcagua y Mapocho, extremos sur del Collasuyu. Boletín del Museo Chileno de Arte Precolombino, en prensa.

Pavlovic, D. y A. Troncoso 2001. Aportes al conocimiento de la ocupación de la Cultura Aconcagua en el curso medio del río Maipo: sitio E-101-3 (TAL 010). Boletín de la Sociedad Chilena de Arqueología 31:48-60.

Pavlovic, D., A. Troncoso, J.C. Hagn y R. Sánchez 1998b. Tal 003-Plaza de Pesaje: Asentamiento de la Cultura Aconcagua en la Confluencia de los Ríos Maipo-Mapocho. Boletín de la Sociedad Chilena de Arqueología 26:22-27.

Pavlovic, D., A. Troncoso, M. Massone y R. Sánchez 1998a. La pequeña casa en la ladera: Blanca Gutiérrez (RML 008), un asentamiento habitacional de la cultura Aconcagua. Boletín de la Sociedad Chilena de Arqueología 25:13-18.

Pavlovic, D., A. Troncoso, R. Sánchez y D. Pascual 2012. Un Tigre en el valle. Vialidad, arquitectura y ritualidad incaica en la cuenca superior del río Aconcagua. Chungara Revista de Antropología Chilena 44 (4):551-569.

Pease, F. 1979. La formación del Tawantinsuyu. Mecanismo de colonización y relación con las unidades étnicas. Histórica 2:97-120.

Planella, M.T. y R. Stehberg 1997. Intervención Inka en un territorio de la cultura local Aconcagua de la zona Centro-Sur de Chile. Tawantinsuyu 3:58-78.

Rostworowski, M. 1988 Historia del Tahuantinsuyu. Instituto de Estudios Peruanos, Lima.
Sánchez, R. 2004. El Tawantinsuyu en Aconcagua (Chile Central) Chungara Revista de Antropología Chilena 36 (2):325-336.

Sánchez, R. 1995. Cultura material, arte, monumentos y cuerpos en el espacio. Prácticas mortuorias del complejo cultural Aconcagua. Actas del XIII Congreso Nacional de Arqueología Chilena. Hombre y Desierto 9: 281-90.

Sánchez, R. y A. Troncoso 2008. Arquitectura, Arte Rupestre y las Nociones de Exclusión e Inclusión. El Tawantinsuyu en Aconcagua (Chile Central). En Lenguajes Visuales de los Incas, editado por P. González y T. Bray, pp. 113-119. BAR International Series 1848, Hadrian Books Ltd., Oxford.

Sanhueza, L. 2001. El aríbalo Inka en Chile Central. Werkén 2:47-69.

Shepard, A. 1956. Ceramics for the Archaeologist. Carnegie Institution of Washington. Washington D.C.

Stehberg, R. 1976a. La Fortaleza de Chena y su Relación con la Ocupación Incaica de Chile Central. Memoria para optar al título profesional de Arqueólogo. Departamento de Antropología, Universidad de Chile, Santiago.

Stehberg, R. 1976b. Notas arqueológicas del cementerio incaico de Quilicura. Santiago, Chile. Noticiario Mensual Museo Nacional de Historia Natural XX (234):5-13.

Stehberg, R. y G. Sotomayor 2012. Mapocho Incaico. Boletín del Museo Nacional de Historia Natural 61:85-149.

Troncoso, A. 2001. Notas y Comentarios: Espacio y Poder. Boletín de la Sociedad Chilena de Arqueología 32:10-23.

Troncoso, A., Letelier, J., Sánchez, R. y P. Larach 2010. Informe final trabajos arqueológicos en sitio Collipeumo. Informe Final proyecto: Caracterización, reparación, conservación y difusión pucara cerro de Collipeumo, región Metropolitana, y trabajos anexos en Cerro Chena. Departamento de Antropología Universidad de Chile, Santiago.

Uribe, M. 2000. La arqueología del Inka en Chile. Revista Chilena de Antropología 15:63-97.

Vázquez, M. 1994. Contextos cerámicos incaicos de Chile Central. Actas del II Taller de Arqueología de Chile Central, pp. 63-97. Santiago.

Williams, V. 2008. Espacios conquistados y símbolos materiales del imperio Inca en el Noroeste de Argentina. En Lenguajes Visuales de los Incas, editado por P. González y T. Bray, pp. 35-57. BAR International Series 1848, Hadrian Books Ltd., Oxford. 